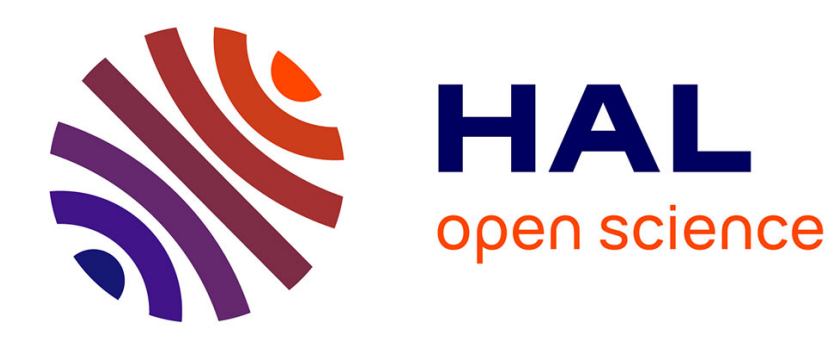

\title{
Le retour à la rue comme support de la gentrification
} Eric Charmes

\section{To cite this version:}

Eric Charmes. Le retour à la rue comme support de la gentrification. Espaces et sociétés (Paris, France), 2005, 122, pp.115-135. halshs-00107415

\section{HAL Id: halshs-00107415 https://shs.hal.science/halshs-00107415}

Submitted on 25 Jan 2007

HAL is a multi-disciplinary open access archive for the deposit and dissemination of scientific research documents, whether they are published or not. The documents may come from teaching and research institutions in France or abroad, or from public or private research centers.
L'archive ouverte pluridisciplinaire HAL, est destinée au dépôt et à la diffusion de documents scientifiques de niveau recherche, publiés ou non, émanant des établissements d'enseignement et de recherche français ou étrangers, des laboratoires publics ou privés. 


\section{Le retour à la rue comme support de la gentrification ${ }^{1}$}

\section{Eric Charmes}

Maître de conférences, Institut Français d’Urbanisme, Université Paris 8

Laboratoire CNRS Théorie des mutations urbaines (UMR Architecture, urbanisme, société)

E-mail : eric.charmes@univ-paris8.fr

Référence : «Le Retour à la rue comme support de la gentrification », Espaces et sociétés, dossier « Le Sens des formes », P. PELLEGRINO (dir.), n 122, 2005, p. 115-135

Version révisée en décembre 2006. Voir aussi E. Charmes, 2006, La rue, Village ou décor? Parcours dans deux rues de Belleville, Grâne, Créaphis, Chapitre 1.

On sait que les regards portés sur les paysages urbains sont des constructions sociales. On sait également qu'ils sont étroitement associés à des classements sociaux (Ledrut, 1968). Mais, dans un ouvrage qui a fait date, Landscapes of Power, paru en 1991, Sharon Zukin est allée au-delà en proposant de lier les mutations sociologiques des espaces urbains aux évolutions de leurs paysages. Elle suggère que ces évolutions sont liées à une concurrence entre groupes sociaux pour l’appropriation des espaces les plus recherchés.

Cet article utilise ce cadre analytique pour étudier le "retour à la rue ». Ce courant urbanistique a incarné à partir des années 1960 le reflux de l’urbanisme moderne. De fait, Le Corbusier et les CIAM avaient fait de la disparition de la rue traditionnelle le symbole du changement qu'ils prônaient. La rue leur a rendu cet honneur en devenant à son tour le symbole de la rupture avec le modernisme. Ce mouvement, amorcé dans les années 1960, s'est élaboré autour de réflexions à la fois sociologiques et paysagères. D’un côté, la rue est apparue comme le lieu où les citadins entrent en contact et où se fonde la culture urbaine. D'un autre côté, elle est apparue comme une forme organisatrice essentielle du tissu urbain traditionnel, au travers de laquelle les bâtiments se lient les uns aux autres et dessinent un

\footnotetext{
${ }^{1}$ L'auteur remercie la lectrice ou le lecteur anonyme pour ses commentaires. L'auteur remercie également le LASMAS pour les données statistiques de l'INSEE qu'il lui a aimablement fournies.
} 
paysage. Le retour à la rue s’est donc placé sous les doubles auspices du lien social et du lien spatial.

La reconnaissance de la valeur paysagère des rues traditionnelles a présidé à l'arrêt, dans les années 1970, de la politique de table rase et de l'urbanisme des tours et des barres au profit d'interventions plus respectueuses des tissus préexistants. La rue est progressivement redevenue un élément structurant des règlements d'urbanisme (Koltirine, 2003). Dans quasiment tous les quartiers anciens, son paysage détermine tant l'implantation que la hauteur des bâtiments.

La reconnaissance de la valeur de la rue comme lieu de vie a été plus longue (Guillerme, 1987), mais c'est à présent un lieu commun de l'urbanisme que de présenter la rue comme un espace essentiel au lien social. Cette reconnaissance ressemble cependant à une victoire à la Pyrrhus. Le retour à la rue s'est en effet largement nourri du constat de la convivialité des rues des quartiers populaires. Or, comme le montre cet article, ce mouvement a été l'un des supports de la gentrification ${ }^{2}$. En réalité, de même que la valorisation du patrimoine des quartiers « historiques » a accompagné le retour dans ces quartiers de populations plus aisées (Bourdin, 1984), le retour à la rue a constitué un système de sens et de valeurs favorable à la gentrification des quartiers populaires et notamment des anciens faubourgs. Bien que mal dotés en patrimoine, ces derniers offrent un paysage urbain traditionnel et des espaces publics très vivants, deux caractéristiques que le retour à la rue a fortement mises en avant. Ce mouvement a ainsi permis à certaines couches moyennes, non seulement de justifier leur présence dans des quartiers populaires, mais aussi de construire une image attrayante de leur environnement.

La présentation qui suit prend appui sur un travail bibliographique ${ }^{3}$ et sur une enquête de terrain ${ }^{4}$. Cette dernière a principalement consisté en la réalisation de plus de vingt visites de rue en compagnie de riverains suivant une démarche librement inspirée de la méthode des parcours commentés (Thibaud, 2001). Les personnes ont été choisies en relation avec le paysage social de la rue. Elles étaient invitées à évoquer leurs pratiques autour de leur espace proche et à commenter le paysage et la vie sociale de leur rue. Le principal intérêt de cette méthode est de mettre la personne en situation : il est plus facile de parler d'un bâtiment lorsqu'il est en face de soi ; par ailleurs, le passage en certains lieux peut réveiller la mémoire d'une rencontre ou d'un incident.

\footnotetext{
${ }^{2}$ Ce terme est utilisé ici dans le sens très large d'élévation de la position sociale des quartiers populaires anciens. Il a été préféré à celui d'embourgeoisement, car cette élévation peut reposer sur l'arrivée de populations postétudiantes aux revenus modérés voire modestes (Pinçon ; Pinçon-Charlot, 2004).

${ }^{3}$ Nous remercions à ce propos Elsa Vivant qui prépare actuellement, à l’Institut Français d’Urbanisme et au laboratoire Théorie des mutations urbaines, une thèse sur la dimension symbolique de la revalorisation des quartiers à Paris.

${ }^{4}$ Cette enquête a été financée par le ministère de l’Environnement dans le cadre du programme « Politiques publiques et paysages » (Charmes, 2003).
} 
Deux rues parisiennes ont particulièrement été étudiées : la rue des Cascades et la rue de l'Ermitage (voir cartes et clichés ci-après). Toutes deux se trouvent sur les hauteurs de Belleville, un ancien faubourg populaire devenu péricentral. Ces deux rues ont l’intérêt de faire partie des premières à avoir bénéficié du regard paysager né avec le retour à la rue. Leur pittoresque leur a en effet permis de bénéficier d'une attention particulière: elles ont notamment fait l'objet dans les années 1970 d’un plan d’aménagement conçu par Antoine Grumbach, l’un des grands noms en France du retour à la rue.

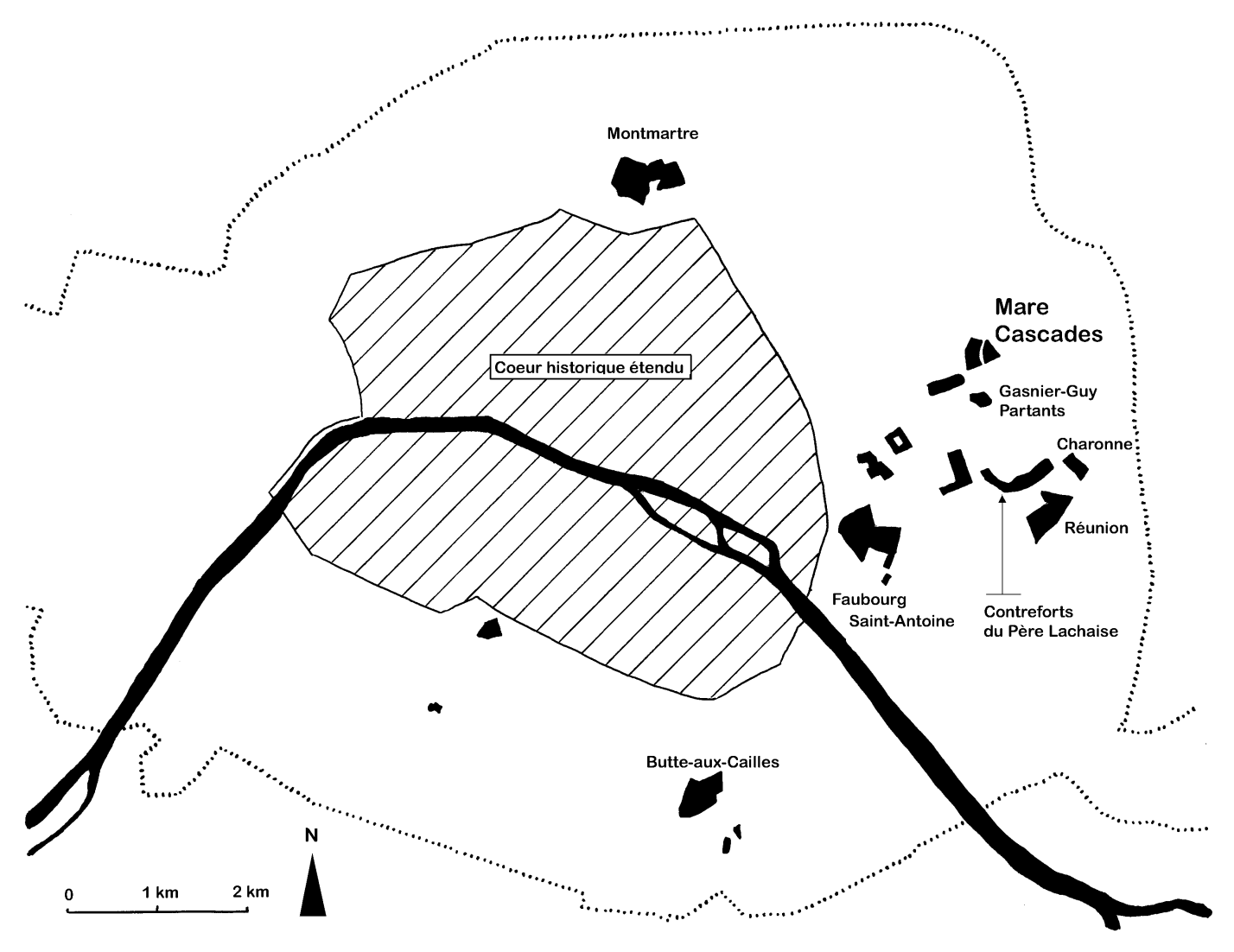

Carte 1 : Localisation des deux rues étudiées

Le trait en pointillé indique les limites de la ville de Paris. Les deux rues étudiées appartiennent au secteur «Mare Cascades ». Les différents secteurs indiqués sont ceux qui ont fait l'objet d'une attention particulière lors de l'élaboration des règlements d'urbanisme qui, à Paris, ont consacré le retour à la rue et la rupture avec le modernisme (Subileau, 1974). Ces règlements ont été mis en œuvre pour la première fois à Paris avec le plan d'occupation des sols de 1977. 
Haut lieu des luttes sociales parisiennes et emblème des quartiers ouvriers, Belleville et, plus particulièrement, les deux rues étudiées connaissent, depuis la fin des années 1980, une incontestable élévation de leur position sociale (Simon, 1995). Le changement social à l'œuvre relève toutefois plutôt de «la gentrification marginale » pour reprendre le terme proposé par Mathieu Van Criekingen et Jean-Michel Decroly (2003). L’arrivée de populations à hauts revenus est en effet considérée dans la littérature comme le parachèvement de la gentrification. Or, à Belleville, le poids de l'habitat social, qui représente $30 \%$ des résidences principales, bloque ce processus (Pinçon; Pinçon-Charlot, 2004). La gentrification de Belleville devrait rester limitée à la présence de populations à revenus moyens (voir tableau 1). Le quartier ressemble ainsi à une mosaïque où des rues préservées et gentrifiées jouxtent des secteurs rénovés avec une forte concentration de logements sociaux.

\begin{tabular}{lcccccc} 
& $\begin{array}{c}\text { Professions } \\
\text { supérieures }\end{array}$ & $\begin{array}{c}\text { Professions } \\
\text { intermédiaires }\end{array}$ & Employés & Ouvriers & Chômage & $\begin{array}{c}\text { Logements } \\
\text { sociaux }\end{array}$ \\
\hline Rues étudiées & 35 & 26 & 25 & 14 & 15 & 10 \\
\hline Belleville & 27 & 24 & 29 & 20 & 16 & 30 \\
\hline Paris & 44 & 23 & 24 & 9 & 12 & 17 \\
\hline
\end{tabular}

Tableau 1 : Caractéristiques socioprofessionnelles de la population active à Paris, à Belleville et dans les rues étudiées

La situation des rues étudiées a été approchée à partir du découpage en IRIS 2000 de l'INSEE. Les chiffres sont indiqués en \% et sont issus du recensement de 1999.

\section{La gentrification comme processus de mutation urbaine}

Le retour à la rue apparaît peu dans la littérature sur la gentrification. Celle-ci propose deux grands schémas explicatifs, initialement opposés, mais qui sont ensuite apparus comme plutôt complémentaires (Bidou, 2003). Certains chercheurs mettent ainsi l'accent sur l'offre de logements et soulignent le bénéfice que les acteurs du marché immobilier peuvent retirer d'une revalorisation d'un quartier central délaissé (Smith, 2003). D’autres insistent plutôt sur l'évolution des pratiques et des demandes sociales (Ley, 2003 ; Florida, 2002). Ils soulignent l'intérêt croissant des classes moyennes pour les ressources culturelles et symboliques des centres urbains. 
Certains des chercheurs de cette dernière tendance se sont penchés sur les transformations graduelles que subissent les quartiers populaires au fil de leur gentrification. Ceux-ci insistent beaucoup sur le rôle des artistes. Ces derniers sont considérés comme les initiateurs, voire comme les moteurs de la gentrification (Ley, 2003). Les artistes sont de fait parmi les premiers à emménager dans les quartiers populaires centraux et péricentraux, puisque beaucoup d'entre eux sont à la fois mal dotés en capitaux économiques et très attirés par les ressources culturelles des centres.

Or l'arrivée d'artistes paraît de nature à changer l'image d'un quartier. Tout d'abord, la présence de cette catégorie sociale garantit aux autres gentrifieurs un équilibre sociologique moins défavorable. Ensuite, et surtout, un quartier investi par les artistes acquiert un statut " branché » et devient progressivement plus attirant pour des personnes plus fortunées, tels que les enseignants du supérieur ou les cadres de la communication et de la publicité. La présence d'artistes permettrait donc une véritable transmutation symbolique. Elle serait au cœur de ce que Sharon Zukin appelle « l’infrastructure critique » de la gentrification (1991).

Les données statistiques sur l'évolution du peuplement des quartiers gentrifiés confèrent une indéniable validité à ce schéma (Ley, 2003). Au demeurant, ce mécanisme semble largement étranger à la conscience des gentrifieurs. Notre enquête suggère que la présence d'artistes intervient très peu, en tant que telle, dans le choix d'autres catégories d'emménager dans un quartier en gentrification. Les personnes rencontrées ont manifesté un intérêt très faible pour les activités des artistes voisins de leur domicile. Ceux-ci ont beau être très nombreux et dynamiques, rares sont les personnes qui ont considéré cette présence comme un atout de leur quartier. Les artistes sont relativement étrangers à leur vie quotidienne. Les personnes qui s'intéressent à l'art le font plutôt au travers du filtre d'une reconnaissance officielle, lorsqu'il est montré dans les musées. Ces derniers sont proches de Belleville, mais sont en dehors de ce quartier.

Ce rapport extrêmement distant aux artistes locaux apparaît très clairement dans les discours tenus sur les journées portes ouvertes organisées chaque année par l'association d'artistes de Belleville. Notre enquête n'a pas valeur de sondage, mais, parmi les personnes que nous avons rencontrées, rares sont celles qui profitent de ces journées pour visiter des ateliers. Quand elles le font, elles cherchent moins à découvrir des œuvres qu'à mieux connaître leur quartier et à visiter des lieux de vie. C'est moins le travail que l'atelier de l'artiste qui les intéressent.

En termes d'ambiance, les atouts de leur quartier mis en avant par les personnes rencontrées renvoient plutôt au retour à la rue. Ce constat ne vaut pas seulement pour Belleville : les premières enquêtes de Catherine Bidou, comme les travaux les plus récents, ont montré que les gentrifieurs construisent l'image positive de leur quartier autour de la double thématique de la convivialité et du mélange social (Bidou, 1984 ; Simon, 1995 ; Urban Studies, 2003). Ils mettent en valeur leur espace résidentiel en insistant, d'une part sur la vie sociale locale, 
d'autre part sur l'enrichissement personnel qu'apporte la mixité sociale. Ce sont précisément les arguments utilisés par l'une des plus grandes figures du retour à la rue, Jane Jacobs.

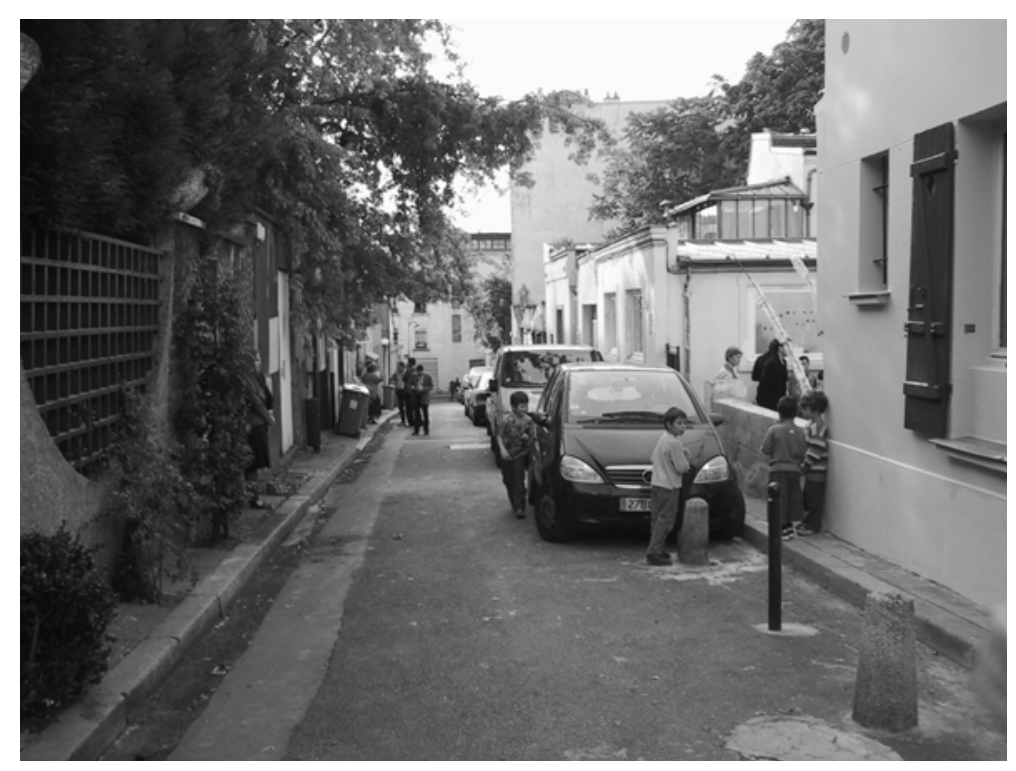

Cliché 1 : La villa de l’Ermitage

Cette rue est attenante à la rue de l'Ermitage (que l'on aperçoit à l'arrière-plan) et est l'une des plus recherchée du secteur par les gentrifieurs.

\section{La construction sociale de l'espace des classes moyennes urbaines}

Dans The Death and Life of Great American Cities (1961), Jane Jacobs dresse une véritable apologie de la rue, présentée comme la matrice de la sociabilité et de la culture urbaine. Cette apologie marque un tournant en ce qu'elle constitue l'une des critiques les plus décisives des utopies du premier vingtième siècle (qu'il s'agisse du modernisme corbuséen ou des citésjardins). Aujourd'hui, cet ouvrage reste une référence majeure de l'urbanisme contemporain. Nombreux sont ceux qui reprennent les arguments de Jane Jacobs pour faire de la rue la forme par laquelle s'incarne la ville, du moins dans ce qu'elle peut avoir de positif pour la civilisation (Gourdon, 2001 ; Rémy, 2004).

A travers la rue, Jane Jacobs oppose l'espace de l'urbanisme moderne, qu'elle juge froid et inhumain, aux villes traditionnelles, dont elle vante tant les stimulations apportées par les rencontres aléatoires que la chaleur et la convivialité. Elle souligne notamment le contraste entre la séparation des fonctions proposée par le modernisme et le mélange fonctionnel des rues, où le commerce se mêle à l'habitat et aux flux piétonniers. Elle relève également que les 
trottoirs et les petits commerces des rues traditionnelles favorisent les rencontres entre les riverains.

Jane Jacobs a ce faisant largement contribué à la diffusion d'une image positive du quartier populaire ancien. Elle a participé à l'élaboration d'un répertoire de sens et de valeurs qui, partout à travers le monde, a favorisé l'emménagement de couches moyennes dans les quartiers populaires des centres urbains. Aujourd'hui, la figure médiatique incontournable de tout débat sur la gentrification qu'est devenue Richard Florida présente The Death and Life of Great American Cities comme l'une de ses principales sources d'inspiration (Florida, 2002) ; il y voit une description précise des principaux attraits des centres et un guide pour les municipalités désireuses d’attirer des populations plus aisées.

En réalité, le retour à la rue a offert un argumentaire opportun pour ceux qui devaient justifier leur emménagement dans des quartiers jusque là délaissés, voire rejetés par les couches sociales aisées ou moyennes. Comme l’a montré Catherine Bidou (1984), les catégories moyennes qui ont émergé avec les Trente Glorieuses se sont heurtées à des difficultés pour trouver un espace urbain qui leur était propre : les quartiers populaires étaient inconfortables et les quartiers bourgeois traditionnels trop onéreux. Dans un premier temps, le périurbain a constitué l’espace privilégié de ces nouvelles couches sociales.

Un changement s'est toutefois rapidement amorcé. Schématiquement, après la phase d'accession au confort matériel qui a caractérisé les décennies de l'après-guerre, une partie des classes moyennes, et notamment les nouvelles générations, ont aspiré à d'autres satisfactions, centrées sur l'expression de soi et l'enrichissement personnel (Bourdin, 1984 ; Ley, 2003). Parallèlement, le style de vie étudiant, et le rapport à la ville auquel il est associé, s'est diffusé bien au-delà de la population étudiante, pourtant elle-même en forte croissance (Van Criekingen ; Decroly, 2003). Ainsi, pour une part significative des classes moyennes, la localisation périurbaine est apparue comme le choix d'individus matérialistes, plus concernés par leur confort matériel et familial que par les stimulations offertes par la vie en centre-ville.

Ces individus à la recherche d'une localisation centrale se sont toutefois heurtés au même obstacle financier que celui qui avait conduit leurs aînés dans le périurbain. L’obstacle était d'autant plus important que le mouvement a été initié par des jeunes adultes et par la fraction intellectuelle des classes moyennes, et donc par des personnes mal pourvues en capitaux économiques. Pour ces personnes, le seul moyen d'habiter au centre était d'entrer dans les quartiers populaires jusque là rejetés.

Il fallait toutefois que ce choix soit mentalement envisageable et publiquement justifiable. Les couches populaires n’ont guère à expliquer leur présence dans un quartier populaire. Ce n'est pas le cas des catégories intermédiaires, dont la présence dans un tel environnement est plus incongrue. Il leur faut régulièrement se justifier devant des membres de leur famille ou devant ceux de leurs amis qui habitent un environnement plus conforme à leur catégorie sociale. Cette justification est d'autant plus nécessaire que le choix d'un quartier populaire fait peser 
(ou peut-être devrait-on dire aujourd'hui faisait peser) le stigmate de la contrainte économique.

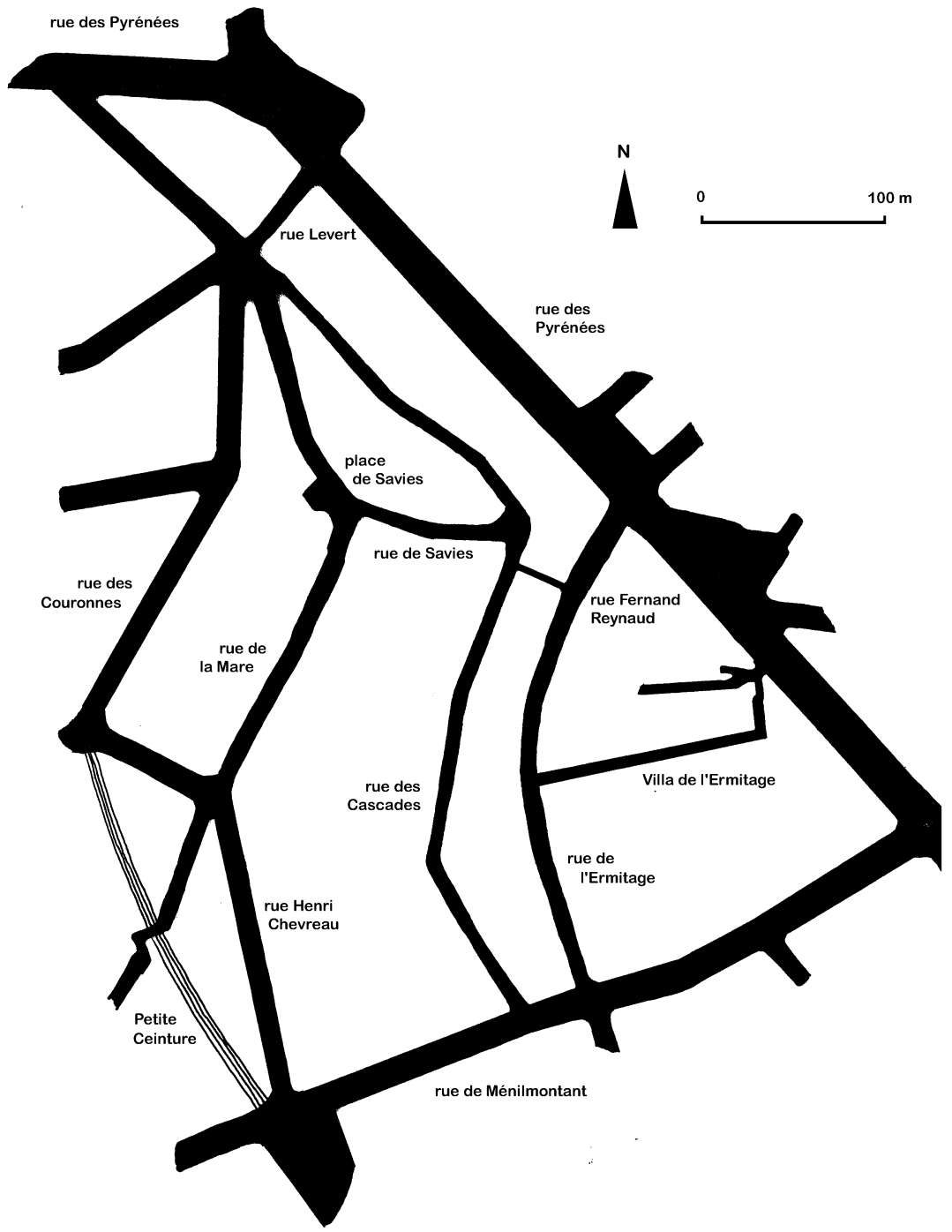

Carte 2 : Les deux rues étudiées et les rues attenantes

L'épaisseur du trait correspond à la largeur réelle des voies. Les deux principales rues passantes et commerçantes du secteur sont la rue des Pyrénées et la rue de Ménilmontant

Ce stigmate initial explique sans doute pourquoi les artistes, et plus largement les catégories sociales proches du monde de la création, sont surreprésentés parmi les pionniers de la gentrification. La bohême jouit en effet d'un certain prestige dans ces milieux : l'absence de capital économique est un signe de pureté intellectuelle, une preuve du refus de toute compromission avec les exigences du marché (Bourdieu, 1992). 
Mais la bohême a ses limites, y compris chez les artistes, et le discours véhiculé par le retour à la rue l'a utilement suppléé. Il a permis de justifier la présence des classes moyennes dans les quartiers populaires en mettant en avant des facteurs d'attraction tels que la convivialité, la solidarité sociale ou encore les stimulations provoquées par le mélange social et la confrontation à l’altérité.

\section{La rue comme paysage humain}

Pour parler comme Pierre Bourdieu, les gentrifieurs semblent faire de nécessité vertu. En donnant une valeur positive au paysage et à l'ambiance des quartiers populaires, ils transmuent un choix relativement contraint en un choix volontaire. L'opportunisme paraît d'autant plus évident que, si certaines personnes rencontrées paraissent sincèrement à l'aise dans l'ambiance bellevilloise, beaucoup avouent facilement qu'elles auraient volontiers résidé dans un quartier moins populaire et qu'elles vivent là simplement parce que le marché immobilier ne leur a pas laissé d’autres choix.

De nombreux sociologues ont effectivement souligné le décalage entre l'image villageoise mise en avant par les gentrifieurs et la réalité de leurs engagements locaux (Bidou, 1984 ; Baudin ; Dupuy, 2001). Alors que, pour les couches populaires, la rue est souvent un espace où l'on vit, pour les classes moyennes, la rue est avant tout un espace que l'on traverse (Charmes, 2003). Pour ces dernières, la sociabilité des couches populaires est plus un spectacle que l'on regarde qu'une scène sur laquelle se déroule une comédie à laquelle on prend part (Bidou, 1984). En réalité, en mettant l'accent sur la convivialité et le mélange social des rues des quartiers populaires, le retour à la rue a avant tout mis en valeur un paysage humain, paysage que les classes moyennes peuvent d'autant plus aisément apprécier que la dureté de la vie quotidienne de ses protagonistes ne les concerne guère.

Dans le prolongement de ce constat, certains sociologues vont jusqu’à dire que les gentrifieurs vivent dans une «bulle » imperméable aux échanges avec les couches populaires (Butler, 2003). La tolérance des gentrifieurs pour le mode de vie des couches populaires atteint de fait très vite ses limites, notamment chez ceux qui ont été les plus contraints par le marché immobilier. L'animation et la chaleur humaine des quartiers populaires ne sont appréciées que tant qu'elles se limitent à une présence humaine autour des boutiques et des étals et à des discussions entre adultes dans la rue. Les autres usages sont loin d'être tous tolérés. Les jeunes qui traînent dans la rue sont particulièrement stigmatisés. Ils suscitent fréquemment un sentiment d’insécurité, voire un mal-être.

Les décalages se révèlent avec une acuité particulière à propos de l'éducation des enfants. A Belleville comme dans de nombreux autres quartiers de ce type (Butler, 2003), les classes moyennes demandent fréquemment une dérogation à la carte scolaire ou ont recours aux 
services du privé. Elles ne changent véritablement de comportement que quand elles deviennent assez nombreuses pour s'immiscer dans la gestion de l'école et imposer leurs normes.

L'ambiguïté des relations entre les discours et les pratiques apparaît également à propos des mutations dont participe la gentrification. En effet, les gentrifieurs sont souvent conscients que leur présence pousse les moins aisés vers des localisations périphériques et contribue à détruire l'ambiance de quartier populaire qu'ils valorisent. Mais l'idéologie de la mixité sociale les aide grandement à surmonter cette contradiction. La valorisation du frottement et du mélange permet en effet d'euphémiser les transformations qu'ils observent: les gentrifieurs peuvent toujours se rassurer en se disant que leur présence sert la cause de la mixité sociale.

Ce n'est ainsi pas un hasard si le mythe d'un Belleville creuset de l'intégration sociale et lieu de côtoiement pacifique des différentes couches sociales est avant tout diffusé par les gentrifieurs (Simon, 1995). Auparavant, les Bellevillois mettaient plus volontiers en avant l'image d'un bastion populaire, haut lieu de la résistance contre la bourgeoisie, avec notamment l'épisode dramatique de la Commune de Paris (Morier, 1994). S’il y avait une mixité, elle était plutôt constituée par un mélange de nationalités.

\section{La rue comme lieu de préparation à l'expérience urbaine}

On ne saurait toutefois réduire la valorisation des ambiances populaires à une sorte de superstructure idéologique servant seulement à habiller des contraintes économiques. L'enthousiasme avec lequel certains gentrifieurs mettent en avant la convivialité et l'ambiance villageoise de leur quartier est trop grand pour n'être que de circonstance. Certes, leurs pratiques quotidiennes les éloignent radicalement des couches populaires. Mais les gentrifieurs ne sont pas indifférents à l'animation que ces dernières donnent aux rues. En réalité, le spectacle de la convivialité les rassure face aux vertiges de la vie urbaine.

La confrontation permanente avec l'altérité, avec des codes de comportement différents et mal maîtrisés, impose de maintenir des bases de stabilité psychologique, pour ne pas dire de sécurité ontologique, comme le propose Anthony Giddens (1991). Les citadins doivent être rassurés sur leur existence sociale et sur la bienveillance du monde environnant. De ce point de vue, quelques signes de sympathie échangés entre voisins soulagent la tension imposée par la vie urbaine. Plusieurs personnes nous ainsi ont parlé avec un bonheur évident des quelques échanges qu'elles ont régulièrement avec d'autres personnes qu'elles croisent dans leur rue. 
Même un simple signe de tête, un bonjour susurré, suffisent à donner le sentiment d'être un habitant et non un simple passant.

L'avantage de la rue populaire, où une sociabilité chaude est mise en scène, est qu'elle permet d'étendre à des anonymes le sentiment de confiance en autrui qui peut naître de ces interactions. Ces dernières prennent en effet place sur le fond de déambulations dans un environnement humain largement anonyme. Même si certains visages sont connus, les passants sont très majoritairement inconnus. Cela concerne particulièrement les rues commerçantes animées dont Jane Jacobs a fait l'apologie, mais cela vaut également pour les rues résidentielles que nous avons étudiées.

Ce mélange de convivialité et d'anonymat fait que, pour ses riverains, la rue peut être un espace d'intermédiation entre le familier et l'inconnu. Les échanges de signes de reconnaissance peuvent être très peu nombreux, la simple reconnaissance visuelle peut ellemême concerner un nombre très limité de personnes, il n'empêche, cela actualise une ambiance générale de convivialité. Pour ténus qu’ils soient, ces signes permettent au sentiment d'être en terrain fiable de s'étendre à des anonymes. Les personnes qui composent la toile de fond des déambulations quotidiennes peuvent bénéficier dans leur ensemble du sentiment de sécurité créé par le croisement régulier de figures familières. Pour peu que des incivilités ne viennent pas troubler l'équilibre qui s'élabore ainsi autour du lien d'habitation, la rue résidentielle peut être un lieu de préparation psychologique à l'expérience de l'espace public urbain.

On comprend alors mieux pourquoi Jane Jacobs a fait simultanément de la rue un espace de sociabilité chaude et un espace de confrontation à l'aléatoire et à l'anonymat. On pourrait voir là une contradiction, tant est établie l'opposition entre l'interconnaissance des quartiers populaires et l'anonymat des espaces publics ${ }^{5}$. La contradiction est cependant plus conceptuelle qu'empirique. En réalité, les représentations mentales construites avec le retour à la rue ont permis de réduire le coût psychologique de l'expérience de la vie urbaine. La rue a pu devenir la forme privilégiée autour de laquelle s'est élaborée une image favorable de la ville, où les expériences rendues possibles par l'anonymat n'éliminaient pas la chaleur des liens sociaux concrets. Pour les classes moyennes, les rues des quartiers populaires se sont avérées particulièrement adaptées à ce mariage: les pratiques y sont apparues à la fois conviviales et exotiques.

\footnotetext{
${ }^{5}$ Comme l'illustrent les ouvrages de synthèse de Lyn Lofland (1998) ou d'Ali Madanipour (2003), cette dichotomie continue de faire référence pour la sociologie urbaine.
} 


\section{Des règles paysagères et des politiques publiques favorables à la gentrification}

Comme nous l'avons souligné, le retour à la rue ne s'est pas seulement incarné par la revalorisation d'un paysage humain, il s'est aussi traduit par une remise à l'honneur des règles d'urbanisme traditionnelles. La rue a bénéficié d'une reconnaissance particulière car elle est au fondement de l'organisation de la ville ancienne : elle dicte tout autant l'orientation et l'implantation des bâtiments les uns par rapport aux autres que leur hauteur, généralement limitée à proportion de la largeur de la rue. Ainsi, pour le promeneur, le paysage urbain traditionnel est essentiellement un paysage viaire, assez ouvert dans les boulevards, plutôt étroit dans les rues résidentielles ou anciennes.

Cette reconnaissance des principes organisateurs de la ville traditionnelle, et plus particulièrement du rôle de la rue, a renforcé la légitimité culturelle du paysage viaire. Ce faisant, elle a accru l'étendue des espaces centraux dotés d'une valeur reconnue. Cela n'est pas anodin : si la reconnaissance de la valeur de l'architecture historique a permis de justifier la présence de gentrifieurs dans des quartiers anciens délaissés, comme le Marais ou comme le Vieux Lyon, cette revalorisation culturelle n'a concerné que des secteurs trop limités par rapport à l'ampleur du mouvement d'expansion des classes moyennes et aisées au sein des centres urbains.

Le retour à la rue a alors opportunément étendu la portée de la reconnaissance culturelle des quartiers anciens aux faubourgs et notamment aux faubourgs populaires ${ }^{6}$. Même si ces derniers n'offrent pas au regard du promeneur des bâtiments à l'architecture exceptionnelle, ils se sont constitués suivant les règles ancestrales que l'urbanisme des CIAM avait bafouées. Si les bâtiments sont sans intérêt particulier, leur organisation d'ensemble est celle de la ville traditionnelle.

Le retour à la rue a également consacré le principe d'une évolution organique de la ville, parcelle par parcelle. Cette politique des petits pas, en rupture avec la table rase prônée par l'urbanisme moderne, s'est avérée plus en phase avec les ressources économiques des gentrifieurs, et notamment des pionniers. Plutôt qu'un logement neuf issu d'une rénovation, ceux-ci pouvaient acquérir à bas prix un appartement ou un local délabré qu'ils pouvaient retaper progressivement, avec l'espoir d'une substantielle plus-value. Il s'agit là d'un point important : de nombreuses études de la gentrification soulignent le poids de l'engagement personnel des gentrifieurs dans la réhabilitation de leur logement, particulièrement dans la phase initiale du processus (Urban Studies, 2003).

\footnotetext{
${ }^{6}$ Une bonne illustration de ce nouveau regard est donnée par l'article rédigé par Jean-Louis Subileau dans le numéro de Paris Projet destiné à présenter et à justifier le plan d’occupation des sols de Paris approuvé en 1977 (Subileau, 1974). Le dossier «Quartiers anciens, approches nouvelles » de cette même revue donne une illustration plus récente de ce regard paysager (Paris Projet, 1998).
} 


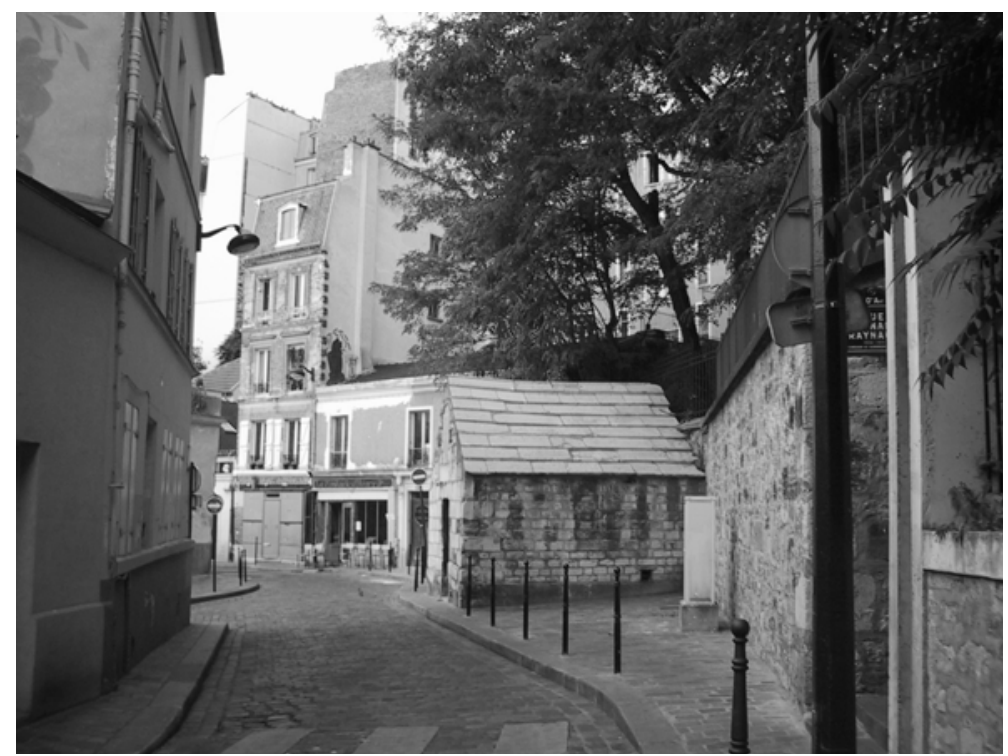

Cliché 2 : Rue des Cascades

Une vue dont le pittoresque explique en partie l'intérêt porté au secteur par l'administration parisienne et par Antoine Grumbach.

\section{L'appui des pouvoirs publics}

Ainsi, de même que les premières étapes du ré-embourgeoisement des centres ont été soutenues par les politiques de préservation du patrimoine mises en place dans le sillage de la loi Malraux de 1962 (Bourdin, 1984), les politiques paysagères inspirées par le retour à la rue ont favorisé l'extension de la gentrification aux faubourgs populaires. Les modifications des règlements d'urbanisme ont été d'autant plus efficaces qu'elles ont été complétées de diverses mesures, telles que les opérations programmées d'amélioration de l'habitat (OPAH), visant à l'éradication de l'habitat insalubre et à la normalisation des quartiers populaires. Certes, alors que la loi Malraux visait seulement la conservation du bâti, les OPAH ont mis l'accent sur le maintien sur place de la population habitante. Mais les garde-fous mis en place n'ont pas empêché les OPAH d'accompagner la gentrification ${ }^{7}$. Tout d'abord, dans un marché aussi tendu que celui des centres urbains, l'amélioration de l'habitat a souvent équivalu à la suppression du rôle de logement social de fait du parc privé dégradé. Par ailleurs, une OPAH contribue à changer la sociologie d'un quartier en attirant des locataires plus jeunes, sous le plafond de ressources au moment de leur emménagement, mais aux meilleures perspectives de carrière que les locataires initiaux. Ensuite, les mécanismes des $\mathrm{OPAH}$ ont fréquemment été contournés : les plafonds de ressource des locataires n’ont pas toujours été respectés et les

\footnotetext{
${ }^{7}$ Pour une vision nuancée des liens entre OPAH et changement social, voir J-Y. Authier, 1993, p. 120 et s.
} 
logements n’ont pas toujours été loués pendant le nombre d'années requis avant d’être reversés sur le marché libre.

Enfin, et surtout, le lancement d'une OPAH est un signe positif pour les investisseurs. Même s'ils ne peuvent pas prétendre aux aides offertes, ce type d'opération leur signale qu'un investissement dans l'amélioration du cadre bâti a toutes les chances d'être rentable. Ils savent que le quartier ne va pas être rasé dans le cadre d'une ZAC, mais qu'il va au contraire prendre de la valeur au fil de l'éradication des taudis. Ce faisant, même s’ils ne sont pas éligibles aux aides, les petits propriétaires se mettent à réhabiliter leur logement et les copropriétés commencent à faire des travaux. Quant aux acteurs plus puissants du marché immobilier, ils guettent les opportunités, attirés par la plus value que laisse espérer un quartier sous-valorisé par rapport à sa localisation (Smith, 2003). Ceux-là peuvent par exemple acquérir une maison ou une ancienne usine désaffectée et construire un nouvel immeuble. Ils peuvent également acquérir un immeuble entier et le remettre totalement à neuf (souvent dans ce cas en bénéficiant d'aides publiques).

L’importance de ces politiques d'aide à la réhabilitation des logements par leurs propriétaires apparaît clairement à l'analyse des situations où elles ont fait défaut. Ainsi, dans le Bas Belleville (autour de la rue Ramponneau et de la rue de Tourtille), le paysage urbain est similaire à celui des rues étudiées mais, moins pittoresque, il a fait l'objet d'une destruction programmée. Cette dernière n'a finalement pas eu lieu, mais la gentrification est restée longtemps bloquée par cette menace de rénovation brutale (au sens de destruction massive de logements anciens et de construction de logements neufs $)^{8}$. En effet, face à une telle incertitude, il était difficile pour les pionniers de la gentrification d'investir dans l'amélioration du cadre bâti du quartier. L'investissement était très risqué et les espoirs de plus-value très faibles. Les perspectives étaient d'autant plus sombres que la mairie de Paris a longtemps pratiqué ce que les associations locales ont qualifié de «stratégie de pourrissement $»^{9}$ : une fois que la ville devenait propriétaire de quelques logements grâce à des préemptions, elle laissait l'immeuble se dégrader et attendait que les locataires et les propriétaires partent pour acheter les logements à un prix réduit.

Leur intérêt paysager a préservé la rue des Cascades et la rue de l’Ermitage de ces pratiques. Leur caractère pittoresque particulièrement marqué ayant retenu l'attention des architectes et des urbanistes qui ont mis en œuvre le retour à la rue à Paris (Subileau, 1974), ces rues ont été placées sous le signe de la réhabilitation (au sens de remise en état des bâtiments anciens) plutôt que sous celui de la rénovation et des démolitions. Elles ont ainsi été les objets d'une OPAH à partir de 1982. Aujourd'hui, il est manifeste que le secteur desservi par les rues des

\footnotetext{
${ }^{8}$ Dans le quartier du Panier à Marseille, le changement social a longtemps été bloqué pour des raisons similaires (Benoît-Guilbot, 1986, p. 135). Mais, à présent, avec le lancement de l'opération Euroméditerranée et avec l'arrivée du TGV, le processus de gentrification du quartier est bel et bien enclenché.

${ }^{9}$ Voir l'opuscule : S. Herszkowicz, Lettre ouverte au maire de Paris à propos de la destruction de Belleville, Paris, Editions de l'encyclopédie des nuisances, 1994.
} 
Cascades et de l'Ermitage est l'un de ceux qui, à Belleville, ont subi la gentrification la plus nette. C'est en tout cas, d'après l'INSEE, le secteur du quartier où la concentration de populations ayant un emploi supérieur est la plus forte.

\section{La valeur symbolique de l'ancien réhabilité}

Tous les secteurs de Belleville n’ont pas subi la stratégie de pourrissement du bas Belleville. Dans plusieurs cas, les rénovations ont rapidement été menées à bien : le secteur Couronnes a ainsi été entièrement remodelé suivant les principes de l'urbanisme moderne avec une dalle, des tours et des barres. Qui plus est, ces rénovations visaient au départ à créer un espace adapté aux exigences des classes moyennes.

Ce but ne semble pas avoir été atteint : une promenade dans Belleville indique clairement que la gentrification est la plus forte dans les rues dont le paysage a été préservé des rénovations et des démolitions massives. Les catégories les plus populaires sont plus représentées dans les secteurs complètement remodelés, et plus particulièrement ceux qui l'ont été suivant les principes de l'urbanisme moderne. Sur les quatorze secteurs avec lesquels l'INSEE a divisé ce qu'elle définit comme le quartier de Belleville, les quatre où la proportion d'ouvriers et d'employés est la plus forte sont les plus marqués par les démolitions et reconstructions des années 1960 à $1980^{10}$. Ceci expliquant cela, ces secteurs rénovés sont aussi ceux où la concentration de logements sociaux est la plus élevée.

Certes, les ménages qui occupent ces logements sont en moyenne plus fortunés que ceux qui occupent le parc ancien dégradé. C'est en effet dans ce parc que l'on trouve les plus fortes concentrations d'ouvriers (et non plus d'ouvriers et d'employés). Mieux, si l'on en croît Patrick Simon, les gentrifieurs qu'il appelle les «transplantés » sont nombreux dans l'habitat social et, plus particulièrement, dans les logements à loyers intermédiaires entre ceux du parc social classique et ceux du parc privé.

Cependant, ces transplantés sont généralement moins bien lotis que les habitants du parc ancien réhabilité. Par ailleurs, si ces ménages contribuent par leur présence à élever le niveau social du quartier, ils ne contribuent pas à la transmutation de l'image du quartier. Au contraire, leur environnement leur apparaissant plutôt sous un angle négatif, ils sont prompts à le critiquer. Ces ménages transplantés jouent donc un rôle secondaire, voire négatif dans la construction du système de sens et de valeurs qui accompagne la gentrification. Ils ne participent pas à la construction d'une image de leur lieu de résidence susceptible d'attirer des

\footnotetext{
${ }^{10}$ Ces secteurs (dits IRIS 2000) sont ceux de la « cité » Piat/Envierges, des Hauts de Belleville, du secteur Couronnes et de la ZAC Bisson/Pali-Kao. Ce dernier aménagement a été réalisé dans les années 1980, mais les trois premiers ont suivi les principes de l'urbanisme moderne.
} 
ménages plus aisés. Ils le font d'autant moins qu'ils vivent dans des ensembles de logements sociaux dont l'image est souvent négative.

Les gentrifieurs qui jouent un rôle décisif dans le changement d'image de Belleville sont avant tout ceux qui occupent le parc privé ancien réhabilité. Les vieux immeubles et les vieilles maisons correspondent mieux aux environnements urbains qu'ils valorisent. Les raisons de cette préférence ne sont pas seulement paysagères, elles sont aussi éthiques : les valeurs d'authenticité et de convivialité dont ils se font les porteurs s'accordent mal avec des rénovations brutales. Ces gentrifieurs acceptent difficilement l'idée de s'installer sur les décombres des anciens faubourgs : comment en effet prétendre rechercher l'ambiance propre aux quartiers populaires en emménageant dans un immeuble neuf construit à la place d'un immeuble de rapport dont les occupants ont été expulsés ? Leur mauvaise conscience est d'autant plus grande que la contestation de l'urbanisme moderne s'est largement nourrie de la dénonciation des déstructurations sociales occasionnées par les rénovations. Plusieurs associations bellevilloises ont d'ailleurs repris ce thème pour lutter contre les projets immobiliers des promoteurs et de la mairie (Lidgi, 2001).

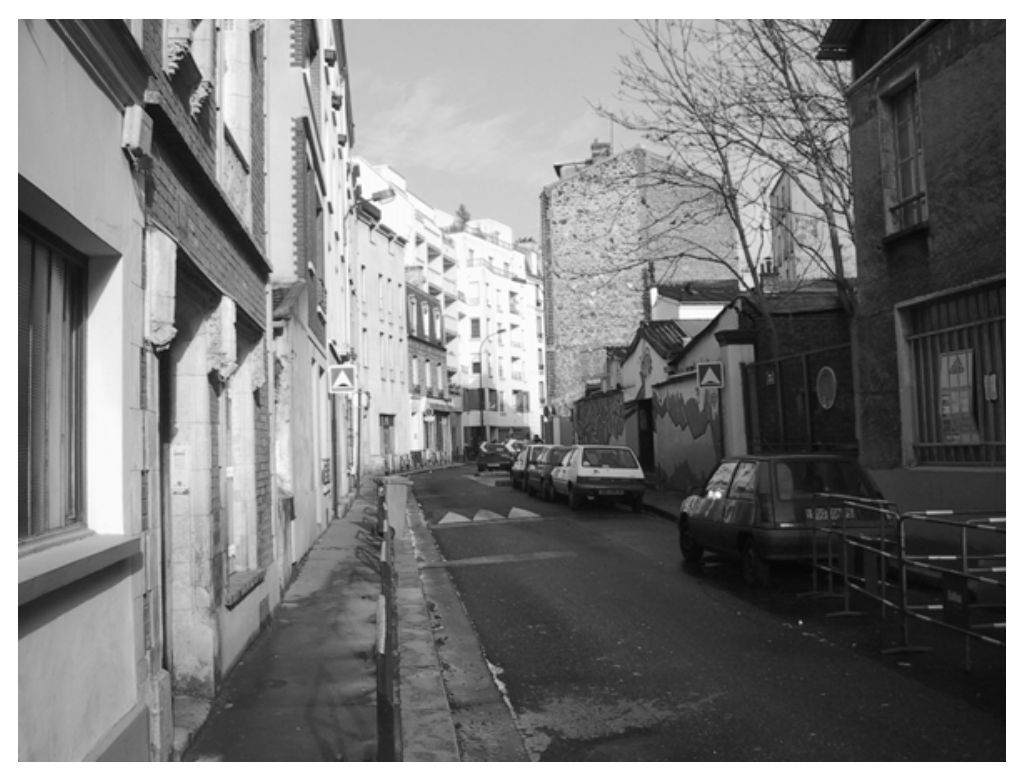

Cliché 3 : Rue de l’Ermitage.

\section{Des rues qui deviennent « pimpantes »}

Quoi qu'il en soit des prévenances des uns et des autres, les classes moyennes expulsent progressivement les ménages populaires hors de Belleville. Les transformations paysagères promues par le retour à la rue sont certes discrètes, mais elles sont plus efficaces que les rénovations brutales de quartiers entiers. Plus que les ensembles d'immeubles neufs ou 
récents, les rues qui gardent leur patine tout en étant partiellement remises à neuf attirent les couches aisées qui tirent la gentrification de Belleville vers un stade plus avancé.

Il suffit pour s'en convaincre d'étudier les propos des gentrifieurs qui souhaiteraient habiter dans des quartiers plus huppés. L'un d'eux, un jeune homme, insiste à plusieurs reprises sur le fait que sa rue est « en complète rénovation » [il parle en réalité des réhabilitations en cours]. Il est visiblement satisfait de ce changement: plus beau ou considéré comme tel par une fraction plus large de la population, ce paysage devient plus facilement valorisant. Les travaux ne progressent au reste pas assez vite à ses yeux : « en matière de rénovation ici, c'est pas encore rénové, c’est une vieille vieille rue ».

On retrouve des propos similaires chez une autre transplantée, habitante de la rue des Cascades âgée d'une trentaine d'années. Commentant un des immeubles situés au dessus du regard Saint-Martin, elle observe avec satisfaction ce qu'elle interprète comme des « essais » préalables à un ravalement. Elle explique : « ils sont en train de prendre en main cet immeuble qui était un peu en perdition ». A propos d'autres réhabilitations, notamment dans la portion nord de la rue des Cascades, elle parle « d'immeubles rattrapés ». Son avis sur le changement apporté par les nombreuses réhabilitations réalisées depuis son arrivée rue des Cascades est plutôt positif. Après avoir égrené les améliorations apportées aux différents bâtiments, elle note « c'est une rue qui dans trois ou quatre ans va être pimpante... C'est partout, c'est dans tous les immeubles, il n’y en aura plus un seul qui sera... [...] C’est complètement transformé ».

Cet enthousiasme n'est pas partagé par les anciens habitants. Ces derniers se sentent évidemment à l'aise avec l'ambiance populaire de leur quartier et ne se sentent pas rassurés par sa remise à neuf, bien au contraire. Celle-ci est ressentie négativement car elle s'accompagne d'une disparition de la population et des activités qu'ils ont côtoyées toute leur vie durant. Les quelques anciens (tous âgés de plus de 70 ans) avec lesquels nous avons parcourus les rues étudiées sont particulièrement frappés par la disparition de la vie ouvrière. Ils égrènent ainsi les fabriques de chaussures et les imprimeurs qu'ils ont vu disparaître un à un. Ils évoquent, avec une pointe de patriotisme déchu, les fabriques insolites, comme celle de baleines de parapluie ou celle d'escargots. Ils sont d'autant plus nostalgiques qu'avec ces fermetures, l'animation diurne de la rue s'est considérablement affaiblie et de nombreux cafés ont à leur tour clos leurs portes.

Pour ces anciens, tout dans le cadre bâti signifie ce qu'ils ne sont plus. Le rapport à l'environnement est marqué par un très fort sentiment de perte d'identité. Quand ils sont encore debout, les vieux bâtiments sont les signes d'un passé révolu et évoquent des activités à présent disparues. Quand ils ont été construits récemment, les immeubles montrent ce qui n'est plus et que la mémoire éprouve plus de difficultés à visualiser, les signes du passé s'étant effacés. Ces évolutions de la rue peuvent être ressenties de manière charnelle (Morier, 1991). Un homme, âgé de plus de quatre-vingts ans et né rue des Cascades, exprime ce sentiment avec une acuité particulière. Arrêté devant un chantier au nord de sa rue, il se 
lamente de l'avancée des réhabilitations et des nouveaux immeubles : « tout va y passer ». Il ressent cela comme une dépossession, ainsi que l'indiquent les propos qu'il tient sur son immeuble: « ils veulent s'accaparer le notre, parce que ça donne jusque dans la rue de la Mare, et il y a beaucoup de terrain tu comprends. La ville de Paris, le jour qu'elle va se décider, elle va donner le paquet, et puis ça y est. Parce que c'est la ville qui achète en premier, elle achète le terrain et puis, après, elle le revend au promoteur, tout ça c'est des histoires de fric et ça marche par millions... Toute la rue va y passer. Tu voies au bout là, ça été construit neuf déjà et petit à petit ça avance [...] petit à petit, petit à petit, ils avancent à mesure qu'ils ont le fric... Parce que comme c'est les impôts qui payent tout ça...». L’impression de financer cette œuvre de destruction ne fait qu'accroître sa colère.

Un autre habitant, âgé de plus de soixante-dix ans et arrivé rue des Cascades dans l'immédiat après-guerre, est nettement moins virulent et souligne que ces transformations étaient nécessaires. Il rappelle en effet à plusieurs reprises que les habitations étaient dépourvues de tout confort : « il n’y avait pas de salle de bain, il n’y avait rien [...] les waters étaient sur le palier, même l'eau était sur le palier » (voire dans la cour). Sur ce plan, les nouvelles constructions ont représenté un progrès : "ça s'est arrangé » juge-t-il. Il reste que lui-aussi entretenait avec son environnement un rapport essentiellement organisé autour des cafés, des usines et des maisons, toutes choses qui ont quasiment disparu du paysage. De fait, chaque immeuble construit après-guerre est pour lui une étape de la transformation de la rue dont il a été le témoin : "ça été bouleversé de tous les côtés » déclare-t-il un peu dépité, une fois parvenu au terme de la visite.

\section{Les paysages du pouvoir}

Pour reprendre les propos de Sharon Zukin (1991), un véritable «paysage du pouvoir » se dessine ainsi autour de la réhabilitation progressive des rues anciennes des faubourgs populaires. Ceux qui sont en phase avec les dynamiques sociales que connaissent ses rues se réjouissent de leur remise à neuf. Ils y voient l'annonce d'un futur radieux. Mais d'autres, comme les anciens habitants, se sentent dépossédés de ce qui a fait leur vie. C’est jusqu’à leur mémoire qu'on leur enlève petit à petit.

Un autre paysage du pouvoir se dessine au travers du type de bâti dans lequel réside les couches populaires. Comme on l'a vu, ces dernières sont surreprésentées dans les secteurs complètement remodelés, et plus particulièrement dans ceux qui l'ont été suivant les principes de l'urbanisme moderne. En même temps, les rues les plus bourgeoisement occupées sont celles qui présentent un paysage traditionnel, relativement préservé des rénovations.

Au travers de cette opposition entre des secteurs paupérisés dont l'architecture évoque les grands ensembles et des rues traditionnelles qui deviennent «pimpantes », le paysage spatial 
s’associe au plus profond des mentalités au paysage social. Un adolescent noir, né de parents immigrés très modestes, a très clairement exprimé cette violence symbolique. Il réside rue des Cascades dans un immeuble de logements sociaux réalisé par Antoine Grumbach. Seulement, sa couleur et sa tenue vestimentaire le classent parmi les occupants naturels de ce qu'il est aujourd'hui convenu d'appeler les « cités ». En conséquence, lors des contrôles d'identité auxquels il est fréquemment soumis, les policiers l'interrogent régulièrement sur sa « cité ». Cela l'exaspère profondément, ainsi que l'indique la réponse qu'il leur fait : «j’habite pas dans une cité, j’habite dans une rue, c'est simplement ce que je leur dis et, à chaque fois, ils s'énervent, mais bon... ». La réponse montre bien à quel point les stigmates sociaux portés par l'image de la cité sont intériorisés par ceux-là mêmes dont on pourrait attendre un contrefeu : habiter une rue est aux yeux de notre interlocuteur le signe d'une position sociale supérieure à ceux qui vivent dans une cité. L'assimilation au monde de la cité qui est opérée par les policiers est vécue comme un acte indu d’infériorisation.

Ce lien entre paysage social et paysage spatial repose en large part sur des stéréotypes. Ainsi, certains grands ensembles ont toujours été bourgeoisement occupés et tous les logements sociaux n’ont pas été construits pendant la vague moderniste. Mais les médias ne s'embarrassent pas de ces détails et, dans les représentations collectives, l'urbanisme moderne est étroitement associé à la pauvreté et aux problèmes sociaux. Cette représentation s’ancre d'autant plus facilement dans les esprits que les grands ensembles sont majoritairement situés dans des espaces de relégation éloignés des centres et que les quartiers au paysage traditionnel sont à l’inverse bien situés et plein d'aménité.

Ce paysage du pouvoir est vivement ressenti par ceux qui vivent dans les grands ensembles paupérisés. Le stigmate social qui pèse sur ces quartiers est difficilement supportable par leurs habitants (Villechaise-Dupont, 2000). Certains y voient une injustice, un manque de reconnaissance de la richesse de leur quartier. Beaucoup d'autres intègrent le regard dominant. Parmi ces derniers, ceux qui le peuvent déménagent, ce qui ne fait qu'aggraver la situation sociale et, en retour, le regard négatif porté sur les grands ensembles. Ceux qui veulent partir, mais qui n'en ont pas la possibilité financière, en conçoivent une amertume particulière. Comme pour se protéger d'un opprobre qu'ils estiment ne pas mériter, ils en attribuent la responsabilité à leurs voisins et notamment aux plus pauvres d'entre eux. Le pas vers le racisme est alors aisé à franchir.

\section{Une gentrification qui restera marginale?}

Cette stigmatisation des grands ensembles de logements sociaux contribue à cantonner dans un stade marginal la gentrification des quartiers qui ont subi d'importantes rénovations. A Belleville, ce sont surtout les populations des «cités » qui maintiennent une atmosphère 
relativement populaire et qui empêche la gentrification d'atteindre un niveau comparable à, par exemple, celui du faubourg Saint-Antoine à Paris (Pinçon \& Pinçon-Charlot, 2004). Ainsi, si le secteur des rues de l'Ermitage et des Cascades est l'un des plus gentrifié de Belleville, il s'agit aussi du secteur où la proportion de logements sociaux est la plus faible : ceux-ci ne représentent que $10 \%$ du parc des résidences principales. Ce sont les secteurs où, à l'inverse, la proportion de logements sociaux dépasse $50 \%$ ou $60 \%$ qui permettent à Belleville de conserver une population d'ouvriers et d'employés. Cette population contribue à imprimer une ambiance populaire aux rues commerçantes du quartier.

On peut s'étonner d'une telle assertion car les populations qui sont venues occuper ces logements sociaux étaient plutôt plus fortunées que celles qui occupaient les taudis détruits. Mieux, si l'on en croît Patrick Simon, les rénovations de l'époque moderne ont contribué à initier la gentrification. Une part significative de ces dernières a tout d'abord été consacrée à la création de copropriétés. Ensuite, même pour la part, très importante, consacrée au logement social, les nouvelles populations appartenaient à des couches plutôt plus aisées que celles qui étaient délogées des logements détruits. On le sait en effet, les populations les plus pauvres n’occupent pas systématiquement les logements sociaux et sont nombreuses dans le parc privé dégradé (Driant, 2002). Cela vaut particulièrement pour les quartiers centraux et cela reste vrai pour Belleville. Les plus fortes concentrations d'ouvriers se trouvent dans les secteurs anciens non réhabilités.

Mais, depuis les premières rénovations, les logements sociaux, et notamment ceux réalisés avant le retour à la rue, sont devenus un frein à l'avancée de la gentrification. En réalité, depuis les balbutiements de la gentrification de Belleville ${ }^{11}$, la position relative de ces logements s'est inversée. Ceux-ci ont progressivement descendu l'échelle des classements sociaux au fur et à mesure que les poches d’habitat privé dégradé disparaissaient. Un à un, les vieux taudis ont été réhabilités et ont laissé la place à des logements remis à neuf ; les immeubles de rapport ont été transformés en copropriétés occupées plus bourgeoisement.

Ainsi, les foyers les plus actifs de la résistance à la gentrification sont aujourd'hui les espaces rénovés brutalement dans les années 1950 à 1970. C’est là que se maintiennent les catégories populaires qui devaient être protégées par le retour à la rue. Destinés au départ aux nouvelles catégories moyennes, ces espaces exercent à présent sur ces dernières un véritable effet repoussoir (le stigmate du grand ensemble). Lorsqu'on leur propose un logement social dans une tour ou une barre, les ménages les plus solvables hésitent à accepter : ils craignent que cela ne leur impose un voisinage indésirable. Cet effet repoussoir ne se limite pas aux tours et aux barres : il déteint sur les rues avoisinantes. En effet, ces immeubles modernes sont les signes d'une présence encore forte et durable des couches populaires et dissuadent les ménages les plus aisés d’acquérir des logements dans les immeubles voisins. On arrive ainsi à ce paradoxe que les foyers de l'ambiance populaire de Belleville (cette ambiance qui a en son

\footnotetext{
${ }^{11}$ Celui-ci a conduit ses enquêtes au tournant des années 1990.
} 
temps contribué à asseoir le retour à la rue) se trouvent aujourd'hui là où l'urbanisme a le plus clairement tourné le dos à la rue.

Belleville n'est pas un cas isolé : la présence de logements sociaux est, d'une manière générale, le principal frein à la gentrification des quartiers populaires (Hamnet, 2003 ; Pinçon \& Pinçon-Charlot, 2004). Le revenu des occupants d'un logement social ne peut excéder un certain niveau et, quand elle est massive, la présence de ces logements interdit aux classes moyennes et aisées de prendre le contrôle des institutions locales (et notamment de l'école). Dans les arrondissements de l'Est parisien, l'effet est d'autant plus marqué que le parc immobilier privé est dominé par les logements de petite taille ${ }^{12}$. Les logements sociaux sont donc fortement surreprésentés dans les appartements familiaux. Les enfants des familles du parc social pèsent alors sur l'ambiance scolaire bien au-delà du poids réel des logements sociaux dans l'ensemble du parc. Or, comme on l'a vu, un établissement scolaire réputé mal fréquenté est un obstacle très fort à l'avancée de la gentrification.

\section{Vers une inversion des signes paysagers favorables à l'architecture moderne?}

En adoptant un point de vue plus prospectif, on peut s’interroger sur la capacité des ensembles des années 1950 à 1970 à résister à la gentrification. D’autres regards pourraient en effet, dans les années qui viennent, conduire à une inversion des signes paysagers. L'importance du pittoresque des rues populaires pourrait notamment refluer devant des valeurs architecturales et paysagères plus «modernes ». Plusieurs évolutions poussent dans ce sens. La légitimation culturelle du parc immobilier des années 1950 à 1970 est ainsi en cours. Si ces immeubles et les paysages qu'ils dessinent sont encore loin d'avoir la faveur du grand public, chez les spécialistes, leur revalorisation paysagère est à l'œuvre. Les architectes sont de plus en plus nombreux à se mobiliser pour la défense des réalisations de cette période lorsqu'elles sont menacées de destruction. L'évolution des normes esthétiques de la photographie contemporaine est un autre signe avant-coureur. Les clichés de plus en plus réputés de Gabriele Basilico font la part belle à l'architecture moderne ${ }^{13}$. Même si le regard de leur auteur se veut en partie critique, ces photographies participent d'une mutation des normes esthétiques défavorable à la ville du passé et à la contextualisation de l'architecture. Il est

\footnotetext{
${ }^{12}$ A Paris, 58 \% des logements comprennent deux pièces ou moins. Le taux est similaire dans le vingtième arrondissement (voir Atelier parisien d'urbanisme, Paris et ses quartiers. Etat des lieux. Eléments pour un diagnostic urbain, Paris, 2001, sur CD-Rom).

${ }^{13}$ Voir par exemple les travaux du photographe Gabriele Basilico.
} 
ainsi significatif que Gabriele Basilico ait été associé à la grande exposition organisée en France autour de Rem Koolhaas ${ }^{14}$.

Parallèlement, les images de vitalité et d'animation propres aux quartiers populaires sont de plus en plus fréquemment associées à la figure des grands ensembles. Il est du reste devenu courant de parler de ces derniers en disant « les quartiers ». Peut-être les « cités » connaîtrontelles un jour l'inversion de regard qu'ont connu les « villages urbains » populaires qui, après avoir été massivement rasés dans les années 1950 et 1960, sont aujourd’hui très recherchés par les gentrifieurs. Une telle destinée pourrait particulièrement concerner les cités les mieux localisées qui, comme la rue aujourd'hui, pourraient bénéficier de l'attractivité des centres urbains.

Le principal obstacle qui se dresse devant un tel changement est la forte proportion de logements sociaux dans les ensembles résidentiels de l'époque moderne. Mais d'une part les bailleurs commencent à mettre une partie de leur patrimoine sur le marché, d'autre part les politiques d'attribution des logements ne sont pas défavorables aux pionniers de la gentrification. La mixité est généralement utilisée par les bailleurs pour justifier une attribution prioritaire des logements des parties dégradées de leur parc aux ménages disposant des meilleurs revenus (Esprit, 2004). Ce faisant, le parc de logements sociaux d'un quartier ou d'une commune en début de gentrification peut être très accueillant pour les ménages pionniers. Si, de surcroît, l'évolution des normes esthétiques amène ces derniers à porter un autre regard sur les grands ensembles, l'effet peut être significatif. Dans ce cas, les derniers bastions de la résistance à la gentrification pourraient tomber.

\section{Pour conclure : la construction sociale du paysage}

Dans les décennies qui ont suivi les années 1960, le regard dominant sur la ville a été orienté par le retour à la rue et, comme on vient de le voir, ce regard est étroitement lié à l'évolution urbaine majeure de cette période : l'investissement des centres et des faubourgs populaires par les classes moyennes et aisées. Les deux volets, paysager et éthique, du retour à la rue ont constitué un système de sens et de valeurs qui a soutenu la gentrification. Les catégories populaires étaient censées être les premières bénéficiaires des politiques mises en place. Elles en ont en réalité été les premières victimes.

Le retour à la rue n'est certes pas une condition suffisante de la gentrification : celle-ci résulte avant tout de la volonté d'une partie des couches moyennes de revenir dans les centres ou de s’y maintenir. Par ailleurs, certains quartiers populaires centraux ou péri-centraux résistent à

\footnotetext{
${ }^{14}$ Voir le catalogue de l'exposition Mutations (2000), p. 440-450. Voir aussi dans ce même ouvrage les clichés de Francesco Jodice, p. 196-206.
} 
la gentrification, par exemple parce que la métropole dans laquelle ils se trouvent n'a pas donné naissance aux couches moyennes gentrifieuses ou parce que la menace d'une rénovation bloque tout investissement dans la réhabilitation des logements. Les configurations des voies urbaines sont également très diverses et toutes ne répondent pas aussi bien aux attentes des gentrifieurs que la rue des Cascades et la rue de l'Ermitage. Certaines voies très passantes ou très marquées par la présence d’industries nuisantes restent délaissées. Mais toutes ces restrictions n'empêchent pas que le retour à la rue ait été et reste un support important de la gentrification.

Ce constat illustre une relation plus générale, bien mise en évidence par Jacques Lucan (1998) : le regard que l'on porte sur une ville est étroitement associé à une manière de l'investir et d'agir sur elle. Le regard hygiéniste a ainsi concordé avec la politique de résorption de l'habitat insalubre. Il a été prolongé en cela par le regard moderniste, qui a également coïncidé avec des politiques de rénovation et d'adaptation de la voiture à la ville. Avant cela, si l'on en croît Henri Bresler (1996), l'annexion par le baron Haussmann des faubourgs de Paris a étrangement correspondu à la construction d'une image "pittoresque » de la banlieue d'alors par les romanciers, les peintres et les graveurs.

On rejoint ici les travaux de Raymond Ledrut (1968) qui avaient montré que la relation entre le spatial et le social passe par le biais d'images construites par la société. De ce point de vue, cet article montre clairement que le pouvoir de la rue ne provient pas seulement des ressources qu'elle apporte à la vie de quartier, mais aussi de l'image qu'elle véhicule. Cette image imprègne d'autant plus les esprits qu'elle s'oppose nettement à celle de grands ensembles.

Belleville n'est pas une exception. D’autres études ont montré que les regards divergents portés sur l'urbanisme moderne et sur la ville traditionnelle ne sont pas sans lien avec les évolutions sociales et les usages des espaces urbains. Dorothée Marchand a ainsi montré que le centre du Havre, rebâti dans un style moderne par Auguste Perret, ne bénéficie pas de la même reconnaissance symbolique et fonctionnelle que le centre historique de Rennes dont le paysage correspond mieux aux canons actuels de l'esthétique urbaine (Marchand, 2003 


\section{Références bibliographiques}

AUTHIER, J-Y. 1993. La Vie des lieux. Un quartier du vieux Lyon au fil du temps, Presses universitaires de Lyon

BAUDIN, G. ; DUPUY, S. 2001. « Le village ambigu. Des voisins de la ville », Les Annales de la recherche urbaine $\mathrm{n}^{\circ} 90, \mathrm{p} .77-84$.

BENOIT-GUILBOT, O. 1986. «Quartiers-dortoirs ou quartiers-villages ? », L'Esprit des lieux. Localités et changement social en France, Paris, Editions du CNRS.

BERGER, M. 2004. Les périurbains de Paris. De la ville dense à la métropole éclatée ?, Paris, Editions du CNRS.

BIDOU, C. 1984. Les aventuriers du quotidien. Essai sur les nouvelles classes moyennes, Paris, PUF.

BIDOU, C. (sous la dir. de) 2003. Retours en ville. Des processus de «gentrification» urbaine aux politiques de « revitalisation » des centres, Paris, Descartes et Cie.

BOURDIEU, P. 1998 [1992]. Les règles de l'art. Genèse et structure du champ littéraire, Paris, Seuil.

BOURDIN, A. 1984. Le patrimoine réinventé, Paris, PUF.

BRESLER, H. 1996. « Découverte moderne. Le pittoresque envahit la ville », dans J. Lucan (sous la dir. de), Paris des faubourgs. Formation, transformation, Paris, Pavillon de l'Arsenal, Picard, p. 148-159.

BUTLER, T. 2003. "Living in the bubble: Gentrification and its "others" in north London », Urban Studies vol. 40, n 12, novembre, p. 2469-2486.

CEFAI, D. ; LAFAYE, C. 2001. « Lieux et moments d'une mobilisation collective. Le cas d'une association de quartier », dans D. Cefaï; D. Trom (sous la dir. de), Les formes de l'action collective. Mobilisations dans les arènes publiques, Paris, Editions de l'EHESS, p. 195-228.

CHARMES, E. 2003. Le retour à la rue : entre paysage et usages. Rue des Cascades et rue de l'Ermitage, Belleville, Paris, rapport de recherche pour le ministère de l'Ecologie et du développement durable, programme " politiques publiques et paysages », ARDU

DRIANT, J-C. 2002. "Le logement social bénéficie-t-il aux ménages à bas revenus ? », Etudes foncières, $\mathrm{n}^{\circ}$ 95, janvier-février, p. 10-13.

Esprit. 2004. « La mixité est-elle une politique ? », mars-avril, p. 121-142.

FLORIDA, R. 2002. The Rise of the Creative Class: and how it's Transforming Work, Leisure, Community and Everyday Life, New York, Basic Books.

GIDDENS, A. 1991. Modernity and Self-Identity. Self and Society in the Late Modern Age, Cambridge, Polity. 
GOURDON, J-L. 2001. La rue. Essai sur l'économie de la forme urbaine, La Tour d'Aigues, Editions de l'Aube.

GUILLERME, A. (sous la dir. de) 1987. La rue n'est pas une route, Colloque européen du laboratoire Théorie des mutations urbaines, Paris.

HAJER, M.; REIJNDORP, A. 2001. In Search of New Public Domain, Rotterdam, Naï Publishers.

HAMNET, C. 2003. "Gentrification and the Mmiddle-class remaking of inner London, 1961-2001 », Urban Studies, vol. 40, n 12, novembre, p. 2422-2423

JACOBS, J. 1994 [1961]. The Death and Life of Great American Cities, Penguin Books.

KOLTIRINE, R. 2003. Règlements et formes urbaines. Etudes comparatives, Paris, Atelier parisien d’urbanisme.

LEDRUT, R. 1968. L'espace social de la ville, problèmes de sociologie appliquée à l'aménagement urbain, Paris, Anthropos.

LEY, D. 2003. "Artists, aestheticisation and the field of gentrification ", Urban Studies vol. $40, \mathrm{n}^{\circ} 12$, novembre, p. 2527-2544.

LIDGI, S. 2001. Paris gouvernance ou les malices des politiques urbaines (J. Chirac/J. Tibéri), Paris, L’Harmattan.

LOFLAND, L.H. 1998. The Public Realm. Exploring the City's Quintessential Territory, New York, Aldine de Gruyter.

LUCAN, J. 1998. « Généalogie du regard sur Paris », Paris Projet n 32-33, p. 20-41.

LYNCH, K. 1960. The Image of the City, Cambridge, Massachusetts Institute of Technology Press.

MADANIPOUR, A. 2003. Public and Private Spaces of the City, Londres, Routledge.

MARCHAND. D. 2003. " Representation of the city and image of the centre in two different urban structures: A modern and a traditional town ", in G. MOSER et alii. Places, People \& Sustainability. Hogrefe \& Huber, Göttingen, p. 11-24.

MORIER, F. (sous la dir. de) 1994. Belleville, Belleville. Visages d'une planète, Paris, Créaphis.

Mutations. 2000. Catalogue de l'exposition, Bordeaux, Arc en Rêve, Centre d'architecture.

PANERAI, P. ; MANGIN, D. 1999, Projet Urbain, Marseille, Editions parenthèses.

Paris Projet. 1998. dossier « Quartiers anciens, approches nouvelles », n 31-32.

PINCON, M. ; PINCON-CHARLOT, M. 2004. Sociologie de Paris, Paris, La Découverte.

REMY, J. 2004. «La Rue et la gare : quel devenir pour la ville ? », Espaces et sociétés $\mathrm{n}^{\circ} 113-114$, p. 261-276. 
ROSSI, A. 2001 [1966]. L’architecture de la ville, Gollion, InFolio éditions.

SIMON, P. 1995. «La société partagée. Relations interethniques et interclasses dans un quartier en rénovation, Belleville, Paris XXe », Cahiers internationaux de sociologie, $\mathrm{n}^{\circ}$ 98, janvier-juin, p. 161-190.

SMITH, N. 2003. «La gentrification généralisée. D’une anomalie locale à la « régénération » urbaine comme stratégie urbaine globale », dans C. Bidou (sous la dir. de), Retours en ville. Des processus de «gentrification » urbaine aux politiques de «revitalisation » des centres, Paris, Descartes et Cie, p. 45-72.

SUBILEAU, J-L. 1974. « Le règlement du P.O.S et le paysage de Paris », Paris Projet n 1314, p. 4-89.

THIBAUD, J-P. 2001. «La méthode des parcours commentés », dans M. Grosjean; JP. Thibaud (sous la dir. de), L'espace urbain en méthodes, Marseille, Editions Parenthèses, p. 79-99.

Urban Studies. 2003. numéro spécial sur la gentrification, vol. 40, nº 12, novembre.

VAN CRIEKINGEN, M. ; DECROLY, J-M. 2003. "Revisiting the diversity of gentrification. Neighbourhood renewal processes in Brussels and Montreal », Urban Studies, vol. 40, n 12, novembre, p. 2451-2468.

ZUKIN, S. 1991. Landscapes of Power. From Detroit to Disney World, Berkeley, University of California Press. 